\title{
Application of Fuzzy Logic and Clustering Techniques for Pavement Maintenance
}

\author{
Amarendra Kumar Sandra ${ }^{1}$. \\ Ashoke Kumar Sarkar ${ }^{2}$
}

Accepted: 28 September 2014 / Published online: 27 May 2015

(C) Springer New York 2015

\begin{abstract}
When a large number of pavement stretches are to be maintained, the decision making becomes complicated and prioritization of individual stretches is not really useful in taking maintenance management decisions. In such situations, grouping or clustering the pavement stretches having similar quantified distress characteristics would be the practical and effective approach. Since a number of distresses are observed on pavements and usually they are being represented in different levels of severity and extent, it gets rather hard to cluster them. The problem becomes further aggravated as the weights of various distresses also play an important role and it is difficult to express them objectively. In this paper, a methodology has been proposed to quantify the pavement distresses and clustering the pavement stretches. The technique has been explained with the aid of a case study carried out in a few selected stretches in the state of Rajasthan, India.
\end{abstract}

Keywords Pavement distresses $\cdot$ Pavement maintenance $\cdot$ Clustering and optimum number of clusters

\section{Introduction}

Besides construction of new highways, proper upkeep of the existing pavements is essential for the economic development of any nation. Inadequate and inappropriate maintenance policies result in grievous financial losses in the strain of ever increasing road user cost (RUC). With the rapid increase in the road construction activities in the less

Amarendra Kumar Sandra amarendra123@gmail.com

Ashoke Kumar Sarkar aksarkar@bits-pilani.ac.in; asarkarbits@gmail.com

1 GMR Infrastructure Ltd.-EPC Division, GMR Hyderabad International Airport Ltd., Pavement Research Centre, Shamshabad, Hyderabad 560409, India

2 Birla Institute of Technology and Science, Pilani, Rajasthan 333031, India 
developed countries, including India, the total funds required for pavement maintenance is continuously increasing and is inadequate. Therefore, there is a need to develop an efficient and methodical system for maintaining the entire road network in an area to the desired serviceability level with the available funds. Pavement Maintenance System (PMS) is an ideal tool in this kind of situation. In any PMS, prioritization of road stretches either individual or groupwise according to their functional condition plays a major part, especially when the funds available for road maintenance are limited $[1,6,7,20]$.

When a large number of pavement stretches are to be maintained in a certain area, it is possible that few stretches have the similar distress characteristics; hence, it is difficult to prioritize within them. In such situations, grouping or clustering the pavement stretches having similar distress characteristics would be the practical and effective approach. In the present study, an attempt has been made to group the pavement stretches according to the extent and severity of distresses. This helps to prioritize a group of pavement stretches instead of a single one so that maintenance measures could be taken accordingly.

\section{Review of Various Clustering Techniques for Grouping of Pavement Stretches}

Clustering is a division of data into groups of similar objects. Each group, called a cluster, consists of the objects that are similar to one another and dissimilar to the objects of the other groups [14]. Many diverse techniques have been developed in order to discover similar groups in large data sets, out of which hierarchical and partitional techniques are being widely practiced $[11,16,19]$.

Hierarchical algorithms create a hierarchical decomposition of the objects into either agglomerative (bottom-up) or divisive (top-down). On one hand, agglomerative algorithms start with each object number as a separate cluster by itself and in turn other objects merge and make a bigger cluster based on the distance between them. The clustering process may terminate when all objects are in a single group or at any other point where the user requires. On the other hand, divisive algorithms follow the opposite strategy. They start with one group of all objects and successively split groups into smaller ones, until each object falls into one cluster, or as desired. The hierarchical algorithms are highly user friendly in the sense that there is no need to specify the number of clusters at the outset. However, it suffers with the major limitation that it is computationally complicated $[16,22]$.

Partitional clustering algorithm constructs partitions of the data, where each cluster forms by minimization of the sum of squared distance from the mean within each cluster. The distance measure usually employed is the Euclidean distance. A close look into the hierarchical and partitional algorithms indicates very clearly that the limitations of one technique are being explained by another technique. Nevertheless, the partitional clustering algorithm needs the number of clusters to be fixed at the beginning itself unlike in the hierarchical algorithm [16]. Though it looks to be a limitation, the flexibility is being offered by partitional algorithm in moving a given pattern from one cluster to another cluster in a dynamically iterative manner. Due to this dynamism, this has been the preferred choice by various researchers for a diversity of applications [14]. It is with this background that these techniques have been used in the present research activity. 
Though a number of specific techniques have been grown within the domain of partitional clustering algorithm, K-means technique has been the preferred choice because of its ease, efficiency, and the simplest algorithms that solve most of the well-known clustering problems $[10,12,15,16,22]$. K-means clustering technique was used to group the pavement sections based on several climate variables for studying their influence on long-term pavement performance [24]. A combination of K-means clustering, analytical hierarchy process, and integer liner programming was used for selecting a group of road projects for maintenance and rehabilitation activities [23]. However, most of the clustering algorithms, including K-means, require the user to set the number of clusters in advance $[9,24]$. Determining the optimum number of clusters is often an ad hoc decision, based on prior knowledge, assumptions, and practical experience [9]. The problem of determining optimum number of clusters in a dataset has been the subject of research [8], and the outcome is still unsatisfactory in this area [21].

After going over the clustering techniques, it has been decided to use K-means clustering technique for grouping the pavement stretches because of its easy application, accuracy, and effective handling of a large amount of data. In all the clustering techniques, it is required to define the number of clusters initially and which is not a correct way to groups the given data set; hence, an approach has been suggested to determine the optimum number of clusters.

\section{Case Study}

\section{Pavement Distress Parameters}

Extent and severity of different pavement distress parameters affect the functional condition of pavements. After visiting a number of road stretches as a part of reconnaissance survey and also by interacting with the field engineers of the Public Works Department (PWD), Rajasthan, India, six different distress parameters was identified as major distresses. They are cracking, raveling (disintegration of asphalt concrete surface due oxidization of asphalt, as a result, dislodgement of aggregate particles), potholes, patching (laying asphalt concrete layer on localized failure), rutting, and edge failure. All of them were expressed in terms of three severity levels, namely, low, medium, and high, and their extents were measured. Each $1 \mathrm{~km}$ length of road was divided into ten stretches of $100 \mathrm{~m}$ length. Data was collected on stretches representing different functional classes of roads such as National Highways (NHs), State Highways (SHs), and Major District Roads (MDRs). The severity levels of the distresses were considered based on the studies carried out by various researchers $[13,17,18]$. The descriptions pavement distresses severities used in this study are shown in Table 1.

Extent of pavement distress parameters such as cracking, raveling, potholes, and patching were measured in terms of area in square meters by encompassing distresses within a regular geometric pattern such as square, rectangle, and triangle. The extents of rutting and edge failures were measured in length along the longitudinal direction. A total of length $39.5 \mathrm{~km}$ (395 uniform sections of $100 \mathrm{~m}$ length) of a 3.5-m-wide lane was considered for data collection. Sample data of pavement distress parameters collected on 20 stretches has been presented in Table 2. For example, in the table, Section 1, RUL 4.7 m means length of the low severity rutting (described in Table 1 ) is $4.7 \mathrm{~m}$ over length of $100 \mathrm{~m}$ and Section $4, \mathrm{CL}=$ $26 \mathrm{~m}^{2}$ represents the area of cracking over a total area of $350 \mathrm{~m}^{2}(100 \times 3.5 \mathrm{~m})$. 
Table 1 Description of flexible pavement distress severity levels

\begin{tabular}{|c|c|c|c|}
\hline \multirow[t]{3}{*}{1} & Cracking & Low & Width of the cracking is less than $3 \mathrm{~mm}$ \\
\hline & & Medium & $\begin{array}{l}\text { Width of the cracking is greater than } 3 \mathrm{~mm} \text { and less } \\
\text { than } 6 \mathrm{~mm}\end{array}$ \\
\hline & & High & Width of the cracking is greater than $6 \mathrm{~mm}$ \\
\hline \multirow[t]{3}{*}{2} & Potholes & Low & Depth of the pothole is less than $25 \mathrm{~mm}$ \\
\hline & & Medium & $\begin{array}{l}\text { Depth of the pothole is more than } 25 \mathrm{~mm} \text { and less } \\
\text { than } 50 \mathrm{~mm}\end{array}$ \\
\hline & & High & Depth of the pothole is more than $50 \mathrm{~mm}$ \\
\hline \multirow[t]{3}{*}{3} & Raveling & Low & $\begin{array}{l}\text { The aggregate or binder has started to wear away } \\
\text { but has not progressed significantly. The pavement } \\
\text { appears slightly aged and somewhat rough }\end{array}$ \\
\hline & & Medium & $\begin{array}{l}\text { The aggregate or binder has worn off and the surface } \\
\text { texture is moderately rough and pitted. Loose } \\
\text { particles may be present and fine aggregate is } \\
\text { partially missing }\end{array}$ \\
\hline & & High & $\begin{array}{l}\text { The aggregate and/or binder have worn off } \\
\text { significantly, and the surface texture is deeply } \\
\text { pitted and very rough. Fine aggregate is essentially } \\
\text { missing from the surface, and pitting extends to a } \\
\text { depth approaching half or more of the coarse } \\
\text { aggregate size }\end{array}$ \\
\hline \multirow[t]{3}{*}{4} & Patching & Low & $\begin{array}{l}\text { Patch has low severity distress of any type including } \\
\text { rutting }<6 \mathrm{~mm} \text {; pumping is not evident }\end{array}$ \\
\hline & & Medium & $\begin{array}{l}\text { Patch has moderate severity distress of any type } \\
\text { or rutting from } 6 \text { to } 12 \mathrm{~mm} \text {; pumping is not evident }\end{array}$ \\
\hline & & High & $\begin{array}{l}\text { Patch has high severity distress of any type including } \\
\text { rutting }>12 \mathrm{~mm} \text {, or the patch has additional different } \\
\text { patch material within it; pumping may be evident }\end{array}$ \\
\hline \multirow[t]{3}{*}{5} & Rutting & Low & Barely noticeable, depth less than $6 \mathrm{~mm}$ \\
\hline & & Medium & Readily noticeable, depth more than $6 \mathrm{~mm}$ less than $25 \mathrm{~mm}$ \\
\hline & & High & Clear effect on vehicle control, depth more than $25 \mathrm{~mm}$ \\
\hline \multirow[t]{3}{*}{6} & Edge Failure & Low & $\begin{array}{l}\text { Appearance of edge step with a few initial cracks on the } \\
\text { bituminous surface along the edge portion of the } \\
\text { carriageway }\end{array}$ \\
\hline & & Medium & $\begin{array}{l}\text { Appearance of edge step with a number of interconnected } \\
\text { high intensity cracks on the bituminous surface along the } \\
\text { edge portion of the carriageway }\end{array}$ \\
\hline & & High & $\begin{array}{l}\text { Permanent loss of part of carriageway and pothole formation } \\
\text { along the edge portion }\end{array}$ \\
\hline
\end{tabular}

\section{Steps Involved in Clustering the Pavement Stretches}

There are three steps involved in determining the optimum number of clusters while developing a maintenance management system for a large road network involving huge amounts of data. They are finding fuzzy prioritization values for pavement stretches using Fuzzy Multi Criteria Decision Approach (FMCDM), grouping of pavement stretches using clustering technique, and determination of optimum number of clusters and its validity. 


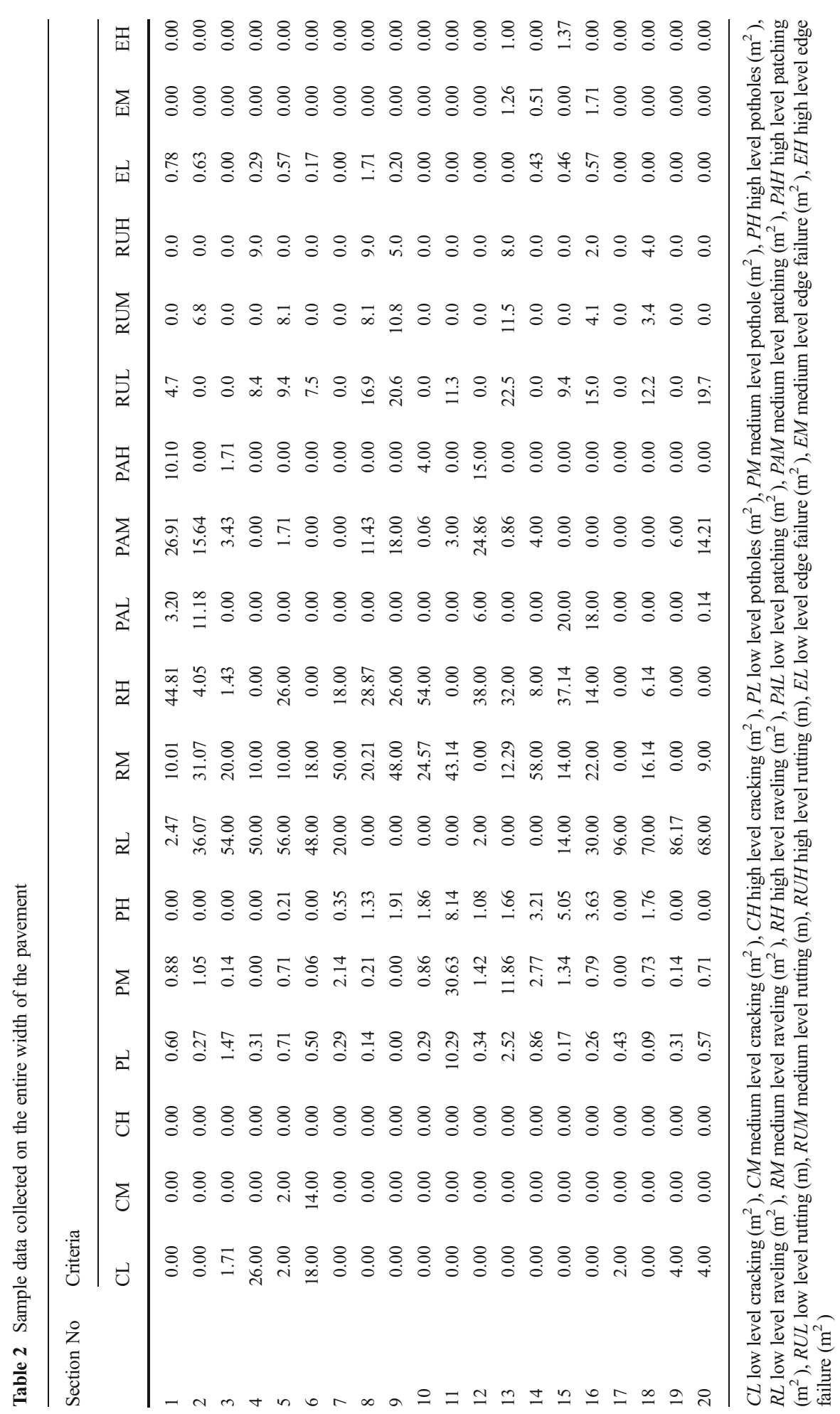




\section{Fuzzy Prioritization Values for Pavement Stretches}

The extent and severity of pavement distresses would have a different impact on the pavement riding quality. Since it is difficult to represent distresses impact in quantifiable terms, normally they are expressed subjectively with the possibility of uncertainty and ambiguity in judgment. In such situations, FMCDM approach provides an ideal option.

Pavement condition data collected on 20 sections of length $100 \mathrm{~m}$ each presented in Table 2 is being used for explaining the prioritization process. There are a few methods available, but the one used by Bandara and Gunaratne [2] and Chen-Tung Chen [3] has been used in this study for its simplicity. Pavement distresses data presented in Table 2 was normalized in the scale of 0 to 100 with respect to the maximum value in the series through a simple normalization using Eq. 1.

$$
\text { Normalized value }=\frac{x_{j i}}{\max \left(x_{j i}\right)} * 100
$$

Where

$x_{j i} \quad$ is the extent of a criteria $C_{j}$ of pavement section $A_{i}$

$\operatorname{Max}\left(x_{j i}\right)$ is the maximum extent value of a criteria $C_{j}$ of pavement section $A_{i}$

These normalized values in the possible range between 0 and 100 thus obtained were given ratings between 1 and 10. The ratings were given with uniform range with the lowest rating being 1 in the range $0-10$ and the highest of 10 in the range 90-100. The ratings were then arranged in a matrix form, named as Rating Matrix $\left(R_{i j}\right)_{N \times M}$ as shown in Eq. 2. In the equation, each row mean pavement sections $\left(A_{1}, A \ldots, A_{N}\right)$ and each column mean criteria or distress parameter. Rating given for distresses of the pavement stretches is presented in Table 3.

$$
\left(R_{i j}\right)_{N \times M}=\left[\begin{array}{cccc}
R_{11} & R_{12} & \cdots & R_{1 M} \\
R_{21} & R_{22} & \cdots & R_{2 M} \\
\vdots & \vdots & \cdots & \vdots \\
R_{N 1} & R_{N 2} & \cdots & R_{N M}
\end{array}\right]
$$

Where

$R_{i j} \quad$ is the real number for the pavement section $A_{i}$ and criteria $C_{j}$

$M \quad$ is the total number of criteria

$N$ is number of stretches

To find the weights of the distresses, the opinions of 20 experts from all over India collected through questionnaire survey were utilized (Table 4). Due to the presence of uncertainty and the weights being subjective in nature, the opinions were sought in five linguistic variables such as "Negligible (N)", "low (L)", "Medium (M)", "High (H)", and "Very High $(\mathrm{H})$ ".

These linguistic variables were converted into weights on a scale of 0 to 1 and then were expressed in Triangular Fuzzy Numbers (TFNs). The weights for various linguistic variables in terms of TFNs were decided by referring the literature for similar examples ([2] and Chen-Tung [3]). TFNs for various linguistic variables are shown in Table 5 and also represented graphically in Fig. 1. 
Table 3 Rating Matrix

$$
\begin{aligned}
& \begin{array}{llllllllllllllllll}
\mathrm{C}_{1} & \mathrm{C}_{2} & \mathrm{C}_{3} & \mathrm{C}_{4} & \mathrm{C}_{5} & \mathrm{C}_{6} & \mathrm{C}_{7} & \mathrm{C}_{8} & \mathrm{C}_{9} & \mathrm{C}_{10} & \mathrm{C}_{11} & \mathrm{C}_{12} & \mathrm{C}_{13} & \mathrm{C}_{14} & \mathrm{C}_{15} & \mathrm{C}_{16} & \mathrm{C}_{17} & \mathrm{C}_{18}
\end{array} \\
& {\left[R_{i j}\right]_{N \times M}=\left[\begin{array}{cccccccccccccccccc|c}
1 & 1 & 1 & 1 & 1 & 1 & 1 & 2 & 9 & 2 & 10 & 7 & 3 & 1 & 1 & 5 & 1 & 1 \\
1 & 1 & 1 & 1 & 1 & 1 & 4 & 6 & 1 & 6 & 6 & 1 & 1 & 6 & 1 & 4 & 1 & 1 & A_{1} \\
1 & 1 & 1 & 2 & 1 & 1 & 6 & 4 & 1 & 1 & 2 & 2 & 1 & 1 & 1 & 1 & 1 & 1 & A_{2} \\
10 & 1 & 1 & 1 & 1 & 1 & 6 & 2 & 1 & 1 & 1 & 1 & 4 & 1 & 10 & 2 & 1 & 1 & A_{3} \\
1 & 2 & 1 & 1 & 1 & 1 & 6 & 2 & 5 & 1 & 1 & 1 & 5 & 8 & 1 & 4 & 1 & 1 & A_{4} \\
A_{5} \\
7 & 10 & 1 & 1 & 1 & 1 & 6 & 4 & 1 & 1 & 1 & 1 & 4 & 1 & 1 & 2 & 1 & 1 & A_{6} \\
1 & 1 & 1 & 1 & 1 & 1 & 3 & 9 & 4 & 1 & 1 & 1 & 1 & 1 & 1 & 1 & 1 & 1 & A_{7} \\
1 & 1 & 1 & 1 & 1 & 2 & 1 & 4 & 6 & 1 & 5 & 1 & 8 & 8 & 10 & 10 & 1 & 1 & A_{8} \\
1 & 1 & 1 & 1 & 1 & 3 & 1 & 9 & 5 & 1 & 7 & 1 & 10 & 10 & 6 & 2 & 1 & 1 & A_{9} \\
1 & 1 & 1 & 1 & 1 & 3 & 1 & 5 & 10 & 1 & 1 & 3 & 1 & 1 & 1 & 1 & 1 & 1 & A_{10} \\
1 & 1 & 1 & 10 & 10 & 10 & 1 & 8 & 1 & 1 & 2 & 1 & 6 & 1 & 1 & 1 & 1 & 1 & A_{11} \\
1 & 1 & 1 & 1 & 1 & 2 & 1 & 1 & 8 & 4 & 10 & 10 & 1 & 1 & 1 & 1 & 1 & 1 & A_{12} \\
1 & 1 & 1 & 3 & 4 & 3 & 1 & 3 & 6 & 1 & 1 & 1 & 10 & 10 & 9 & 1 & 8 & 8 & A_{13} \\
1 & 1 & 1 & 1 & 1 & 4 & 1 & 10 & 2 & 1 & 2 & 1 & 1 & 1 & 1 & 3 & 4 & 1 & A_{14} \\
1 & 1 & 1 & 1 & 1 & 7 & 2 & 3 & 7 & 10 & 1 & 1 & 5 & 1 & 1 & 3 & 1 & 10 & A_{15} \\
1 & 1 & 1 & 1 & 1 & 5 & 4 & 4 & 3 & 10 & 1 & 1 & 7 & 4 & 3 & 4 & 10 & 1 & A_{16} \\
1 & 1 & 1 & 1 & 1 & 1 & 10 & 1 & 1 & 1 & 1 & 1 & 1 & 1 & 1 & 1 & 1 & 1 & A_{17} \\
1 & 1 & 1 & 1 & 1 & 3 & 8 & 3 & 2 & 1 & 1 & 1 & 6 & 3 & 5 & 1 & 1 & 1 & A_{18} \\
2 & 1 & 1 & 1 & 1 & 1 & 9 & 1 & 1 & 1 & 3 & 1 & 1 & 1 & 1 & 1 & 1 & 1 & 1 \\
2 & 1 & 1 & 1 & 1 & 1 & 8 & 2 & 1 & 1 & 6 & 1 & 9 & 1 & 1 & 1 & 1 & 1
\end{array}\right] A_{19}}
\end{aligned}
$$

Where

$C_{1}$ low severity cracking (CL), $C_{2}$ medium severity Cracking (CM), $C_{3}$ high severity cracking $(\mathrm{CH}), C_{4}$ low severity potholes (PL), $C_{5}$ medium severity potholes (PM), $C_{6}$ high severity potholes $(\mathrm{PH}), C_{7}$ low severity raveling $(\mathrm{RL}), C_{8}$ medium severity raveling $(\mathrm{RM}), C_{9}$ high severity raveling $(\mathrm{RH}), C_{10}$ low severity patching (PAL), $C_{11}$ medium severity patching (PAM), $C_{12}$ high severity patching (PAH), $C_{13}$ low severity rutting (RUL), $C_{14}$ medium severity rutting (RUM), $C_{15}$ high severity rutting (RUH), $C_{16}$ low severity edge failure (EL), $C_{17}$ medium severity edge failure (EM), $C_{18}$ high severity edge failure (EH)

The experts opinion obtained in the form of linguistic variables as presented in Table 4 were then converted into TFNs using Table 5. To normalize the differences existing in expert opinion, a simple average of the fuzzy number for each distress criteria was calculated and the corresponding weights were worked out. Fuzzy weights for all criteria can be expressed in the form of the column matrix as shown in Eq. 3.

$$
\widetilde{W}_{j}=\left[\begin{array}{c}
\widetilde{w}_{1} \\
\widetilde{w}_{2} \\
\vdots \\
\widetilde{w}_{M}
\end{array}\right]
$$

Where $\widetilde{w}_{1}, \widetilde{w}_{2} \ldots \ldots \ldots ., \widetilde{w}_{M}$ are the fuzzy weights for all criteria expressed in TFNs, i.e., $\widetilde{w}_{j}=\left(\widetilde{w}_{j 1}, \widetilde{w}_{j 2}, \widetilde{w}_{j 3}\right) \quad \forall j=1,2,3 \ldots \ldots . M$

Fuzzy prioritization value $\left(\widetilde{p}_{i}\right)$ was then calculated by multiplying the rating matrix with the fuzzy weight matrix and added up separately for each section, 


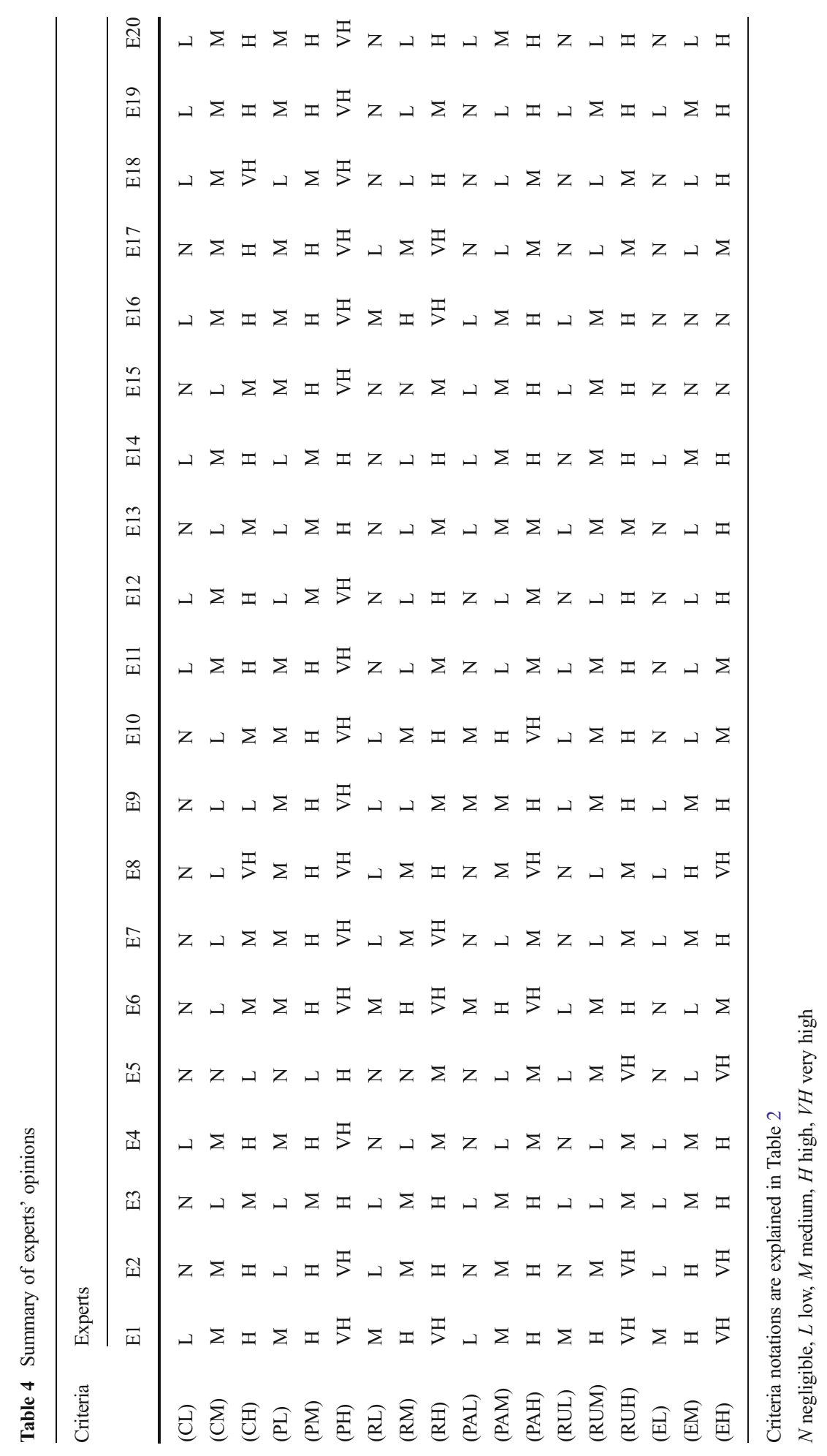


Table 5 Triangular fuzzy numbers (TFNs) for linguistic variables

Linguistic variable

Triangular fuzzy number

$\begin{array}{ll}\text { Negligible } & (0,0,0.1) \\ \text { Low } & (0,0.1,0.3) \\ \text { Medium } & (0.3,0.5,0.7) \\ \text { High } & (0.7,0.9,1) \\ \text { Very high } & (0.9,1,1)\end{array}$

which has been shown in Table 6. This process is expressed mathematically as follows:

$$
\widetilde{P}_{i}=\sum_{j=1}^{M} R_{i j} \otimes \widetilde{w}_{j}, \quad \forall i=1,2, \ldots \ldots . . N \text { and } \forall j=1,2,3 \ldots \ldots . M
$$

Where

$R_{i j}$ is the real number for the pavement section $A_{i}$ and criteria $C_{j}$

$\widetilde{w}_{j}$ is the fuzzy weight matrix

\section{Clustering of Pavement Stretches Using K-Means Clustering Technique}

The objective of the K-means is to find the partition of the data, which minimize the squared error or the sum of the squared distances between all the points and their respective cluster centers. In other words, K-means minimize the intra-cluster distance. The algorithm is composed of the following steps [14, 22]:

1. Choose $\mathrm{K}$ initial cluster centers.

2. Assign each data point to the group that has the closest center by calculating the distance between all the centers and the data points.

3. After assigning all the data points, recalculate the positions of the $\mathrm{K}$ centers.

4. Repeat second and third steps until the $\mathrm{K}$ centers will no longer move.

Fuzzy prioritization values for all the stretches which are shown in Table 6 are then grouped with the help of K-means clustering technique. For this work, a suitable code was developed in MATLAB and used for clustering the pavement stretches. Flowchart for K-means clustering technique is shown in Fig. 2.

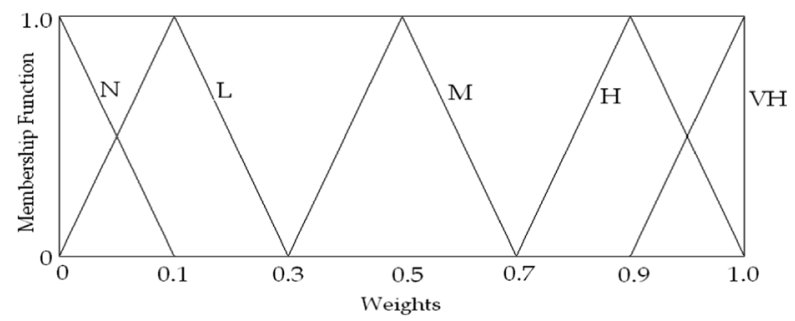

Fig. 1 TFNs for different weights 
Table 6 Fuzzy prioritization values for all the stretches
Stretch

Fuzzy prioritization value

$\widetilde{p}_{1}$

$\widetilde{p}_{2}$

$\widetilde{p}_{3}$

$\widetilde{p}_{4}$

$\widetilde{p}_{5}$

$\widetilde{p}_{6}$

$\widetilde{p}_{7}$

$\tilde{p}_{8}$

$\widetilde{p}_{9}$

$\widetilde{p}_{10}$

$\widetilde{p}_{11}$

$\widetilde{p}_{12}$

$\widetilde{p}_{13}$

$\widetilde{p}_{14}$

$\widetilde{p}_{15}$

$\widetilde{p}_{16}$

$\widetilde{p}_{17}$

$\widetilde{p}_{18}$

$\widetilde{p}_{19}$

$\widetilde{p}_{20}$
$(16.22,23.07,30.23)$

$(9.09,14.42,21.46)$

$(7.40,11.02,15.41)$

$(11.40,16.54,23.07)$

$(10.14,15.34,21.93)$

$(7.96,12.93,20.04)$

$(9.09,13.21,17.84)$

(17.93, 26.01, 35.31)

$(17.55,25.87,35.39)$

$(14.74,19.85,24.43)$

$(21.85,29.91,37.81)$

(17.98, 25.01, 31.69)

$(25.00,35.20,45.56)$

$(11.39,16.19,21.54)$

(20.67, 27.52, 34.57)

(15.06, 22.04, 31.03)

$(6.01,8.98,12.94)$

$(11.48,16.36,22.16)$

$(6.41,9.68,14.01)$

$(7.34,11.64,17.80)$

\section{Finding the Optimum Number of Clusters (ONC)}

In K-means clustering technique, it is necessary to give the number of the groups to be made as an input. Since it is not possible to fix the optimum number of clusters arbitrarily for any given data set, in the present study, an iterative algorithm was developed to find the number of clusters.

Step 1: Applying the code developed in MATLAB for K-means clustering technique, the fuzzy evaluation values were clustered into homogeneous groups.

Step 2: Cluster centroid was calculated using Eq. 5.

$$
\overline{x_{j}}=\sum_{i=1}^{n} x_{i j} / n \quad \forall j=1, \ldots, 5
$$

Where

$j \quad$ is the number of variables in one data point

$n \quad$ is the number of data points in a cluster

$\overline{x_{j}} \quad$ is the $j$ th column centroid

$x_{i j}$ is the data point in $i$ th row and $j$ th column

$\forall \quad$ represents for all 


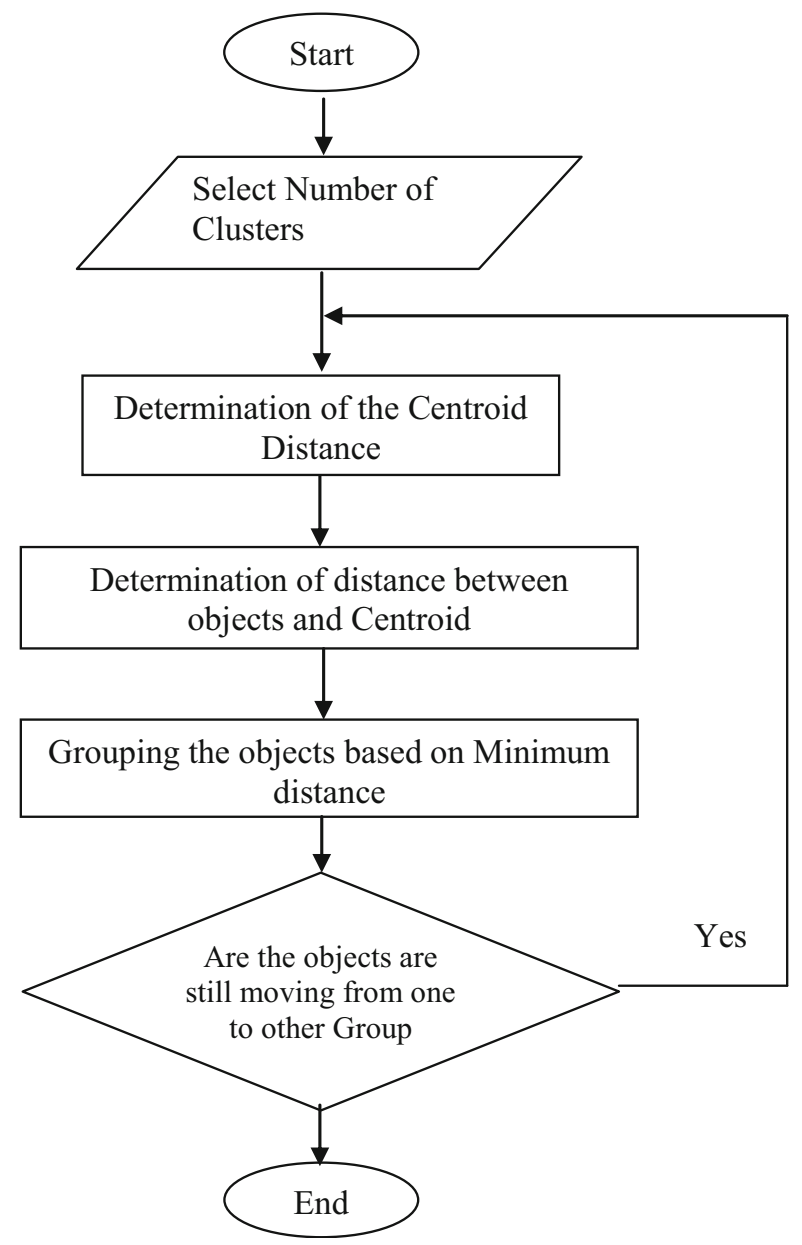

Fig. 2 Flow chart for K-means clustering technique

Step 3: For all the individual clusters in a cluster group, the distance between data points and its cluster centroid (also known as intra-cluster distance) was computed using Eq. 6.

$$
D_{i}=\sqrt{\sum_{i=1}^{n}\left(x_{i j}-\bar{x}_{j}\right)^{2}} \quad \forall i=1, \ldots, n \text { and } \forall j=1, \ldots, 5
$$

Where $D_{i}$ is the distance of $i$ th row in a cluster from its centroid and the remaining parameters are as explained in Eq. 5.

Step 4: The average of all the distances from each data point and its corresponding cluster centroid was computed for all the individual clusters in a chosen cluster group using Eq. 7. Further, the weighted averages of the distances of the each cluster group were 
Table 7 Distances and weighted average distances of different cluster group

\begin{tabular}{|c|c|c|c|c|c|c|c|c|}
\hline \multirow{2}{*}{$\begin{array}{l}\text { Number } \\
\text { of cluster } \\
\text { groups }\end{array}$} & \multicolumn{8}{|c|}{$\begin{array}{l}\text { Number of data points in each cluster (average of distances of the data points from their respective } \\
\text { cluster centers) }\end{array}$} \\
\hline & 1 & 2 & 3 & 4 & 5 & 6 & 7 & $\begin{array}{l}\text { Weighted average } \\
\text { distance of cluster } \\
\text { group }\end{array}$ \\
\hline 1 & $20(12.15)$ & & & & & & & 12.15 \\
\hline 2 & $8(5.36)$ & $12(4.67)$ & & & & & & 4.946 \\
\hline 3 & $3(5.12)$ & $6(3.85)$ & $11(4.21)$ & & & & & 4.238 \\
\hline 4 & $7(3.71)$ & $1(0.00)$ & $5(2.39)$ & $7(2.40)$ & & & & 2.736 \\
\hline 5 & $3(1.70)$ & $4(2.57)$ & $5(2.39)$ & $7(2.40)$ & $1(0.00)$ & & & 2.2 \\
\hline 6 & $3(1.70)$ & $1(0.00)$ & $4(2.00)$ & $4(2.57)$ & $7(2.27)$ & $1(0.00)$ & & 1.96 \\
\hline 7 & $2(0.87)$ & $1(0.00)$ & $4(2.00)$ & $7(2.27)$ & $4(2.04)$ & $1(0.00)$ & $1(0.00)$ & 1.8 \\
\hline
\end{tabular}

calculated using Eq. 8. The outcome of this exercise is presented in Table 7.

Where

$$
\bar{D}_{k}=\frac{1}{n} \sum_{i=1}^{n} D_{i} \quad \forall k=1, \ldots, K \text { and } \forall n=1, \ldots, K
$$

$K \quad$ is the total number of clusters in a cluster group

$k \quad$ is the individual cluster number in a cluster group

$n$ is the total number of data points in individual cluster

$$
K_{\mathrm{WA}}=\frac{\sum_{k=1}^{K} \bar{D}_{k}{ }^{*} n_{k}}{\sum_{k=1}^{K} n_{k}}
$$

Where

$K_{\mathrm{WA}} \quad$ is the weighted average distance of the cluster group

Step 5: A graph was plotted between weighted average distances of all the cluster groups and the number of cluster groups as shown in Fig. 3. It has been noted that the weighted average distance decreases as the number of the clusters increases.

The optimum number of clusters is observed to be the point where the increase in the number of clusters does not result in any appreciable reduction in the distances. It may be observed from Fig. 3 that the distance of cluster groups rapidly decrease up to number five and then the gradient of the curve becomes comparatively flat and there is not much change 


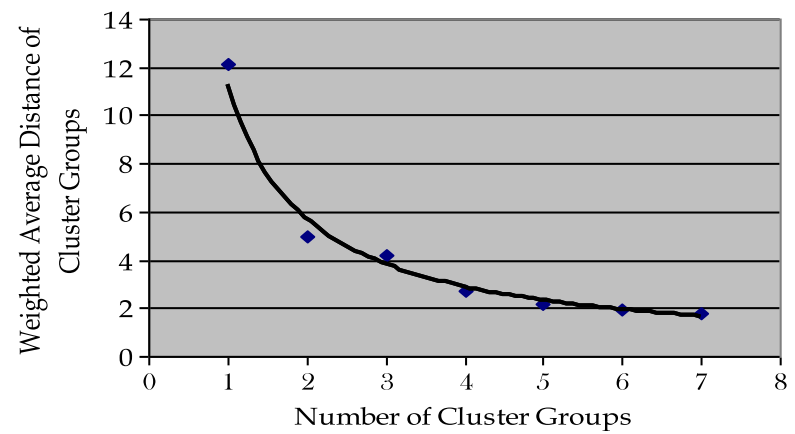

Fig. 3 Plot between number of cluster group and weighted average distance of cluster group

in the value from 6 to 7 and further. Thus, ONC in this may be considered to be six. A sample calculation to determine the weighted average distance of cluster group has been shown in the Appendix.

\section{Validation of the Optimum Number of Clusters}

Though an algorithm has been developed and presented for finding the $\mathrm{ONC}$, it was decided to cross-check its acceptability through the existing cluster validity techniques. Popular techniques viz. Dunn's and Davies-Bouldin indices, as detailed in the following paragraphs, were chosen for validation.

Dunn's Validity Index This technique $[5,16]$ is based on the idea of identifying the cluster sets that are compact and well separated. The main goal of Dunn's validity index is to maximize the inter-cluster distances (i.e., separation) while minimizing intracluster distances (i.e., increase compactness). The Dunn's validation index (DV) can be computed using Eq. 9. The number of clusters, which maximizes the DV, is considered as the ONC.

$$
\mathrm{DV}=\min _{k=1, \ldots, K}\left\{\min _{l=k+1, \ldots . K}\left\{\frac{d\left(c_{k}, c_{l}\right)}{\max _{a=1, \ldots, K}\left\{d^{\prime}\left(c_{a}\right)\right\}}\right\}\right\}
$$

Where

$d\left(c_{k}, c_{l}\right)$ is the distance between clusters $c_{k}$ and $c_{l}$ in the cluster group

$d^{\prime}\left(c_{a}\right) \quad$ is diameter of the clusters in the cluster group $a$

$K \quad$ is the number of clusters in a group

Davies-Bouldin Validity Index This index $[4,16]$ is a function of the ratio of the sum of intra-cluster scatter to inter-cluster separation. The ratio is small if the clusters are compact and far from each other. Consequently, Davies-Bouldin index (DB) will have a small value for ONC. The DB index can be computed using Eq. 10.

$$
\mathrm{DB}=\frac{1}{K} \sum_{k=1}^{K} \max _{\substack{l=1, \ldots ., K \\ k \neq l}}\left(\frac{d^{\prime}\left(c_{k}\right)+d^{\prime}\left(c_{l}\right)}{d\left(c_{k}, c_{l}\right)}\right)
$$


Table 8 Dunn's and DB validity indices

\begin{tabular}{lll}
\hline Number of clusters & Dunn's index & DB index \\
\hline 2 & 1.70 & 0.93 \\
3 & 0.94 & 1.33 \\
4 & 1.69 & 0.72 \\
5 & 1.32 & 0.65 \\
6 & 2.29 & 0.49 \\
7 & 1.94 & 0.56 \\
\hline
\end{tabular}

All the parameters are as explained in Eq. 9.

Using Eqs. 9 and 10, DV and DB values have been calculated for a different number of clusters and presented in Table 8 .

It can be observed that the Dunn's index gives maximum value at six numbers of clusters while the DB index, which functions based on minimization criteria, also provides the ONC as six. It can be recalled here that the ONC obtained from the algorithm used in the present study was also six. Hence, it was decided that the optimum number of clusters for the data considered is 'six' and the technique proposed to find the ONC is acceptable.

\section{Utility of Clustering in Pavement Maintenance Decision}

The clusters may be prioritized for making decision on pavement maintenance management. In a road network, each cluster may contain a large number of stretches, and it may be necessary to cluster them further. The methodology suggested in this paper may be applied for this purpose. This will help to prioritize clusters with small and manageable number of pavement stretches.

\section{Conclusions}

- For a huge road network, it is necessary to cluster the pavements having similar distress characteristics for effective decision making in pavement management system.

- Level of discomfort to the road users by pavement distresses places an important role in identifying the sections which needs prior attention. Since it is difficult to represent them in quantifiable terms, usually they are expressed subjectively with the possibility of uncertainty. In such situations, FMCDM approach provides an ideal option.

- The approach suggested in clustering of a large number of pavement stretches into a manageable number of clusters is an ideal option for stagewise maintenance of pavement stretches depending on the funds available.

\section{Appendix}

\section{Calculation of Weighted Average Distance of Cluster Groups}

In the present study, a total of 20 pavement stretches were considered as an example of clustering. As discussed in "Finding the Optimum Number of Clusters (ONC)" section, 
Table 9 Data points in first group

\begin{tabular}{lllll}
\hline Sample number & \multicolumn{2}{l}{ Fuzzy evaluation values } & $\begin{array}{l}\text { Intra-cluster } \\
\text { distance }\end{array}$ \\
\hline 1 & 16.215 & 23.07 & 30.23 & 6.825564 \\
2 & 17.93 & 26.01 & 35.305 & 1.370885 \\
3 & 17.545 & 25.865 & 35.385 & 1.776309 \\
4 & 21.845 & 29.91 & 37.81 & 4.933227 \\
5 & 17.98 & 25.01 & 31.69 & 4.077818 \\
6 & 25 & 35.2 & 45.56 & 14.60829 \\
7 & 20.665 & 27.52 & 34.565 & 1.885166 \\
8 & 15.06 & 22.035 & 31.03 & 7.480824 \\
Cluster centroid & 19.03 & 26.8275 & 35.19688 & \\
\hline
\end{tabular}

based on the fuzzy prioritization, values of all 20 stretches were clustered into homogeneous groups using K-means clustering technique. The following steps explain the calculation of weighted average distance when data points were made into two groups. The same procedure has been followed for more number of groups also.

Step 1: A total of 20 stretches were clustered into two groups. Out of the 20 data points eight are falling in first group as presented in Table 9 and 12 are falling in second group as shown in Table 10.

Step 2: Cluster centroid (average of the data points in the column) and the intracluster distance (distance from the centroid to data point) was calculated using the expression given in Eq. 6. Cluster centroid and intra-cluster distances are also appended in the respective tables.

Step 3: Average of each cluster group (average of all intra cluster distances)

Table 10 Data points in second group

\begin{tabular}{lllll}
\hline Sample number & Fuzzy evaluation values & $\begin{array}{l}\text { Intra-cluster } \\
\text { distance }\end{array}$ \\
\hline 1 & 9.085 & 14.415 & 21.455 & 2.170685 \\
2 & 7.4 & 11.02 & 15.41 & 5.249276 \\
3 & 11.395 & 16.54 & 23.065 & 5.001045 \\
4 & 10.135 & 15.34 & 21.925 & 3.05412 \\
5 & 7.96 & 12.925 & 20.04 & 1.798006 \\
6 & 9.085 & 13.205 & 17.84 & 1.688328 \\
7 & 14.735 & 19.845 & 24.43 & 9.510686 \\
8 & 11.385 & 16.185 & 21.54 & 3.776937 \\
9 & 6.01 & 8.98 & 12.935 & 8.739572 \\
10 & 11.475 & 16.36 & 22.16 & 4.307206 \\
11 & 6.405 & 9.675 & 14.005 & 7.414167 \\
12 & 7.335 & 11.635 & 17.8 & 3.385122 \\
Cluster centroid & 9.367083 & 13.84375 & 19.38375 & \\
\hline
\end{tabular}


has been calculated. First group and second group averages are 5.36 and 4.67 , respectively.

Step 4: Weighted average of the clustered group $((8 * 5.36+12 * 4.67) / 20=4.94)$ has been calculated using the expression given in Eq. 8 .

\section{References}

1. Alsugair, A.M., Al-Qudrah, A.A.: Artificial neural network approach for pavement maintenance. J. Comput. Civ. Eng. ASCE 4(4), 249-255 (1998)

2. Bandara, N., Gunaratne, M.: Current and future pavement maintenance prioritization based on rapid visual condition evaluation. J. Transp. Eng. ASCE 127(2), 116-123 (2001)

3. Chen, C.-T.: A fuzzy approach to select the location of the distribution center. Fuzzy Set. Syst. 118, 65-73 (2001)

4. Davies, D.L., Bouldin, D.W.: A cluster separation measure. IEEE Trans. Pattern Anal. Mach. Intell. 1(2), 224-227 (1979)

5. Dunn, J.C.: Well separated clusters and optimal fuzzy partitions. J. Cybern. 4, 95-104 (1974)

6. Farhan, J., Fwa, T.: Incorporating priority preferences into pavement maintenance programming. J. Transp. Eng. 138(6), 714-722 (2012)

7. Fwa, T.F., Tan, C.Y., Chan, W.T.: Road-maintenance planning using genetic algorithms. II: analysis. J. Transp. Eng. ASCE 120(5), 710-722 (1994)

8. Halkidi, M., Batistakis, Y., Vazirgianni, M.: On clustering validation techniques. J. Intell. Inf. Syst. 17(23), 107-145 (2001)

9. Hamerly, G.J., Elkan, C.: Learning the K in K-Means. Department of Computer Science and Engineering, University of California, San Diego (2003). http://books.nips.cc/papers/files/nips16/NIPS2003_AA36.pdf

10. Hamerly, G.J.: Learning structure and concepts in data through data clustering. PhD thesis submitted to Department of Computer Science, University of California, San Diego. (http://cs.baylor.edu/ hamerly/ papers/thesis.pdf) (2003)

11. Han, J. and Kamber, M.: Data mining: concepts and techniques, second edition, Elsevier, Morgan Kaufmann Publishers. (http://www.cs.uiuc.edu/homes/hanj/bk2/toc.pdf) (2006)

12. Ilampiray, P.: Efficient resource utilization of web using data clustering and association rule mining. J. Theor. Appl. Inf. Technol. 37(2), 211-216 (2012)

13. IRC-82: Code practice for maintenance of bituminous surfaces of highways. Indian Road Congress, New Delhi (1982)

14. Jain, A.K., Murthy, M.N., Flynn, P.J.: Data clustering: a review. ACM Comput. Surv. 31(3), 264-323 (1999)

15. Jayanta, B.: Online adaptive hierarchical clustering in a decision tree framework. J. Pattern Recognit. Res. 2, 201-229 (2011)

16. Mahamed G. H. O.: Particle swarm optimization methods for pattern recognition and image processing. PhD thesis submitted to Faculty of Engineering, Built Environment and Information Technology, University of Pretoria, Pretoria. (http://upetd.up.ac.za/thesis/available/etd-02172005-110834/unrestricted/ 00front.pdf) (2005)

17. Miller, J.S and William. Y.B.: Distress identification manual for long-term pavement performance program. Report submitted to Office of Infrastructure Research and Development, Federal Highway Administration, Publication No.FHWA-RD-03-031. http://www.fhwa.dot.gov/publications/research/ infrastructure/pavements/ltpp/reports/03031/03031.pdf) (2003)

18. NCHRP: Automated pavement distress collection techniques, National Cooperative Highway Research Program, Synthesis 334. Transportation Research Board, Washington D.C. (2004) http://onlinepubs.trb. org/onlinepubs/nchrp/nchrp_syn_334.pdf

19. Pradeep, R. and Subha, S.: A survey of clustering techniques. Int. J. Comput. Appl. (0975-8887) Vol. 7No.12, (2010)

20. Reddy, B.B. and Veeraragavan: Priority ranking model for managing flexible pavements at network level. Technical paper published in 62nd Annual Session of Indian Roads Congress (IRC), 8th-11th January, Kochi, India. (http://mail.mahapwd.com/technicalpapers/Volume\%20623/paperno478.htm) (2002) 
21. Rosenberger, C., Chehdi, K.: Unsupervised clustering methods with optimal estimation of the number of clusters: application to image segmentation. Proc. Int. Conf. Pattern Recog. 1, 1656-1659 (2002)

22. Turi, R.H.: Clustering based colour image segmentation. $\mathrm{PhD}$ thesis submitted to Monash University, Australia. (http://www.csse.monash.edu.au/ roset/thesis/thesis.pdf). (2001)

23. Wu, Z. and Flirtsch, C.W.: Optimal selection of pavement maintenance and rehabilitation programme; 7th International Conference on Managing Pavement assets, June 24-28, 2008, Calgary, Alberta. (http:// pavementmanagement.org/ICMPfiles/2008098.pdf). (2008)

24. Yuhong, W.: Improving pavements with long-term pavement performance: products for today and tomorrow. Federal Highway Publication, Report No. FHWA-RD-03-049.(http://www.fhwa.dot.gov/ publications/research/infrastructure/pavements/ltpp/03049/paper5.cfm). (2005) 